\title{
Self-induced inverse spin Hall effect in ferromagnets: Demonstration through nonmonotonic temperature dependence in permalloy
}

\author{
O. Gladii, ${ }^{1, *}$ L. Frangou, ${ }^{1}$ A. Hallal, ${ }^{1}$ R. L. Seeger, ${ }^{1}$ P. Noël, ${ }^{1}$ G. Forestier, ${ }^{1}$ S. Auffret, ${ }^{1}$ M. Rubio-Roy, ${ }^{1}$ P. Warin, ${ }^{1}$ \\ L. Vila, ${ }^{1}$ S. Wimmer, ${ }^{2}$ H. Ebert, ${ }^{2}$ S. Gambarelli, ${ }^{3}$ M. Chshiev, ${ }^{1}$ and V. Baltz ${ }^{1, \dagger}$ \\ ${ }^{1}$ Univ. Grenoble Alpes, CNRS, CEA, Grenoble INP, SPINTEC, F-38000 Grenoble, France \\ ${ }^{2}$ Universität München, Department Chemie, Butenandtstr. 5-13, D-81377 München, Germany \\ ${ }^{3}$ Univ. Grenoble Alpes, CEA, SYMMES, F-38000 Grenoble, France
}

(Received 29 August 2019; published 6 November 2019)

\begin{abstract}
We investigated the self-induced inverse spin Hall effect in ferromagnets. Temperature $(T)$, thickness $(t)$, and angular-dependent measurements of transverse voltage in spin pumping experiments were performed with permalloy films. Results revealed nonmonotonous $T$ dependence of the self-induced transverse voltage. Qualitative agreement was found with first-principles calculations unravelling the skew scattering, side-jump, and intrinsic contributions to the $T$-dependent spin Hall conductivity. Experimental data were similar whatever the material in contact with permalloy (oxides or metals), and revealed an increase of produced current with $t$, demonstrating a bulk origin of the effect.
\end{abstract}

DOI: 10.1103/PhysRevB.100.174409

\section{INTRODUCTION}

The generation of a spin current and its further conversion to a charge current have attracted considerable attention, facilitating advances in basic physics along with the emergence of closely related applications in the field of spintronics [1,2]. The electronic transport regime considers spins carried by conduction electrons, in contrast to the magnonic transport regime which refers to excitation of localized magnetic moments [3,4]. Electronic spin current can be considered to occur through two distinct mechanisms: drift "spin-polarized" current, when spins are carried by conduction electrons drifting due to the effect of an electric field; and diffusive "pure" spin current, which is caused by diffusion of conduction electrons bearing majority spin and minority spin in opposite directions. In the case of diffusive spin current, diffusion results from non-equilibrium conditions creating a spin imbalance. This imbalance can be triggered by several mechanisms including distinct densities of states at the interface between materials of different types [e.g., ferromagnetic metals (F) and nonmagnetic metals (NM)], and transfer of angular momentum between phonons, photons, and electrons [2]. In this context, an electrical current can be converted to a spin current and vice versa as a result of the spin-orbit interaction (SOI), which links the spin and the orbital angular momentum of an electron. As a result of SOI, a flow of charges (spin) causes transverse spin (charge) to accumulate [5]. One of the related effects of this phenomenon, known as the inverse spin Hall effect (ISHE) [6,7], is commonly used to study SOI in NMs inserted into archetypal F/NM bilayers. In some of these studies, a spin current is pumped from the F spin injector at

\footnotetext{
*olga_gladiy@ukr.net

†vincent.baltz@cea.fr
}

resonance $[8,9]$, and the ISHE ensures spin-charge conversion in the NM [10]. The contribution of the F to spin-charge conversion can be difficult to distinguish from that of the NM, and spin-charge conversion arising from the $\mathrm{F}$ is frequently neglected in experiments [11-18]. However, as we will further discuss below, in some cases spin-charge conversion in the $\mathrm{F}$ may prevail and need to be carefully considered. Some experimental studies indicated that self-induced charge current can be generated at room temperature $(T)$, e.g., in $(\mathrm{Ga}, \mathrm{Mn}) \mathrm{As}$ [14], NiFe [13,18], Co [15], and Fe [15] ferromagnets at resonance. Self-induced charge current can be considered to occur through two distinct mechanisms: magnonic chargepumping (MCP) [14,18-20], or ISHE. While both mechanisms are triggered by spin-orbit interactions in ferromagnets, they are fundamentally different. References [14] and [18] report experimental investigation at room temperature of the reciprocal spin-orbit torques (SOT) phenomenon known as MCP [19], or inverse SOT (ISOT) [20]. This effect is dictated by lack of spatial inversion symmetry (bulk or structural). In contrast, Refs. [13] and [15] propose a mechanism for the origin of this spin current based on spin-dependent scattering at the different interfaces. More specifically, when magnetic moments precess, the angular momentum of $3 d$ electrons is transferred to $4 s$-conduction electrons leading to a spinpolarized current in the F. Spins then flow in a diffuse manner due to non-uniform magnetization, which has been ascribed to asymmetric spin relaxation at the various interfaces. SOI in the $\mathrm{F}$ further ensures spin-charge conversion via the ISHE. Experimental data indicated a conversion efficiency of about $1 \%$ for $\mathrm{NiFe}$ [13].

In this study, we investigated the self-induced ISHE in single permalloy thin films when brought to resonance. Most importantly, our results demonstrated the bulk origin of the effect. Our experimental data revealed the self-induced transverse charge current to have a nonmonotonous $T$ dependence. 
(a)

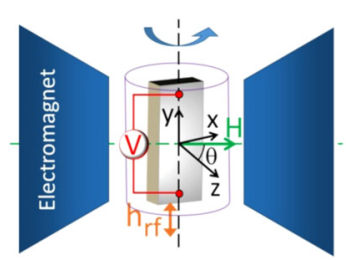

(c)
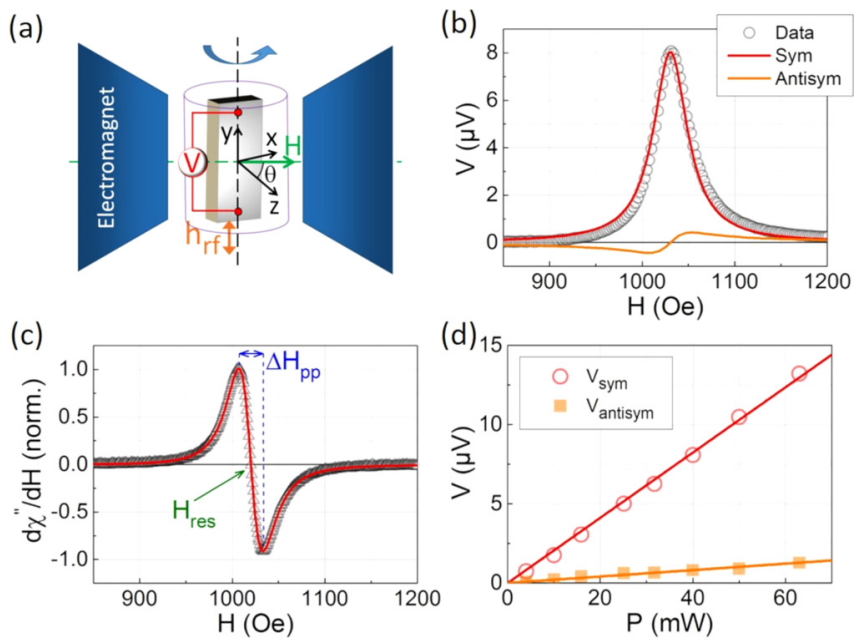

(d)

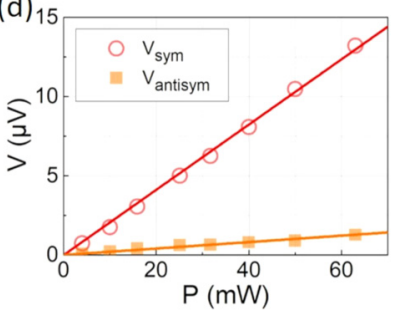

FIG. 1. (a) Representation of the experiment design. (b) Representative data showing $H$ dependence of $V$, as measured for a $\mathrm{Si} / \mathrm{SiO}_{2} / / \mathrm{Cu}(6) / \mathrm{NiFe}(8) / \mathrm{Cu}(3) / \mathrm{Al}(2) \mathrm{Ox}(\mathrm{nm})$ stack at $95 \mathrm{~K}$, when $\theta=+90^{\circ}$ and $P=40 \mathrm{~mW}$. (c) Corresponding input power $(P)$ dependence of $V_{\text {sym }}$ and $V_{\text {antisym }}$. (d) Corresponding differential absorption spectra $\left(d \chi^{\prime \prime} / d H\right.$ vs $H$ ). The lines in (b) and (d) were fitted to the data, see text. The lines in (c) correspond to linear fits of the data constrained to pass through $(0,0)$.

This finding was corroborated by the results of first-principles calculations describing the various contributions to the $T$ dependent spin Hall conductivity.

\section{EXPERIMENTS}

The stacks used were: $/ / \mathrm{Cu}(6) / \mathrm{NiFe}\left(\mathrm{t}_{\mathrm{NiFe}}=8 ; 12\right.$; $16 ; 24 ; 32) / \mathrm{Cu}(3) / \mathrm{Al}(2) \mathrm{Ox}(\mathrm{nm})$ multilayers. Stacks were deposited at room $T$ by sputtering on $\mathrm{Si} / \mathrm{SiO}_{2}(500) / /$ substrates at a pressure of $2.3 \times 10^{-3}$ mbar under argon. The $\mathrm{NiFe}$ layer was deposited from a $\mathrm{Ni}_{81} \mathrm{Fe}_{19}$ (at. \%) permalloy target. A 2-nm-thick Al cap was deposited to form a protective $\mathrm{Al}(2) \mathrm{Ox}$ film after oxidation in air. The sample dimensions were: $l=2.46 \mathrm{~mm}$ and $w=0.46 \mathrm{~mm}$. Both sides of the samples were connected to electrodes using Al-wire bonding.

Spin pumping experiments [Fig. 1(a)] were conducted in a continuous-wave electron paramagnetic resonance spectrometer. The sample was fitted with a three-loop-two-gap resonator operating at $9.6 \mathrm{GHz}[21,22]$. An input power $P$ of $40 \mathrm{~mW}$ was used, corresponding to an excitation magnetic field of $h_{\mathrm{rf}} \sim$ $0.5 \mathrm{Oe}$, along the $y$ direction. The precise value of this field was determined for each data point by measuring the quality factor of the cavity $Q$ and using the equation given for our MS5 resonator: $h_{\mathrm{rf}}^{2}=4 P Q / 500$. The further normalization by $h_{\mathrm{rf}}^{2}$ thus takes into account any variability of the rf-to-material coupling. A dc bias field $(\boldsymbol{H})$ was simultaneously applied at an angle $(\theta)$ with respect to the sample normal $(\boldsymbol{z})$. For each angle tested, the amplitude of $\boldsymbol{H}$ was scanned across the resonant condition for the NiFe layer's magnetization $(\boldsymbol{M})$. The corresponding electric potential difference $(V)$ induced along the $y$ direction as a result of spin pumping and spincharge conversion was then recorded. The field-sweep rate was $14 \mathrm{Oe} \mathrm{s}^{-1}$. A typical $V$ versus $H$ spectrum is shown in Fig. 1(b). The symmetric (sym) and the antisymmetric (anti-
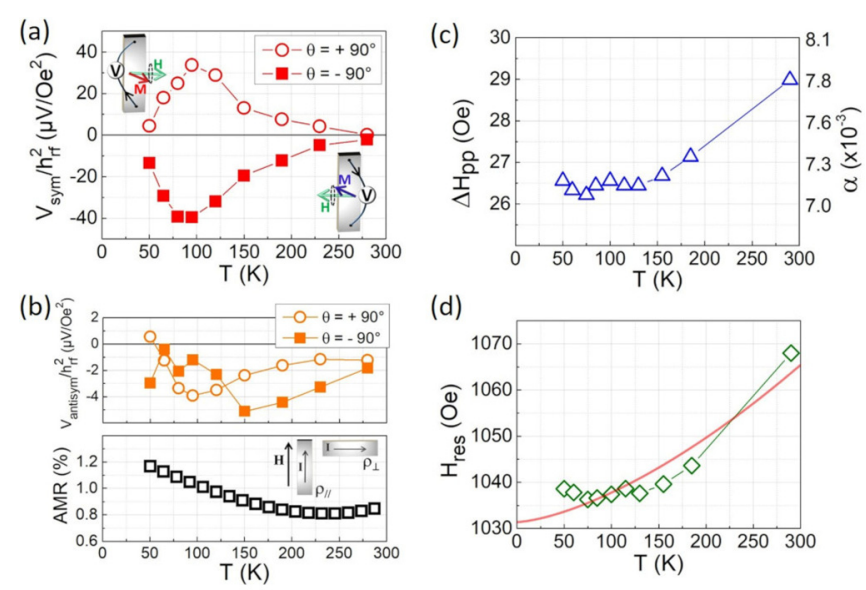

(d)

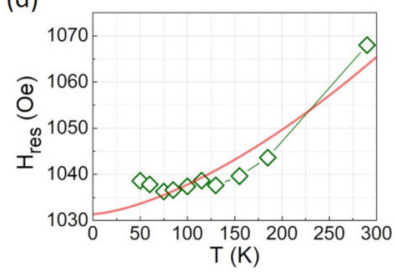

FIG. 2. (a) $T$ dependence of the symmetric contribution, $V_{\text {sym }}$ to $V$, normalized by "the microwave power" $\propto h_{\mathrm{rf}}^{2}$. Data measured for a $\mathrm{Si} / \mathrm{SiO}_{2} / / \mathrm{Cu}(6) / \mathrm{NiFe}(8) / \mathrm{Cu}(3) / \mathrm{Al}(2) \mathrm{Ox}(\mathrm{nm})$ stack, when $\theta= \pm 90^{\circ}$. (b) $T$ dependences of the antisymmetric contribution, $V_{\text {antisym }}$ plotted along with the AMR. [(c) and (d)] $T$ dependences of $\Delta H_{\mathrm{pp}}$ (and corresponding $\alpha$ ) and $H_{\mathrm{res}}$. The line was obtained using the Kittel model.

sym) contributions were disentangled by fitting data using the following equation:

$$
\begin{aligned}
V= & V_{\text {sym }} \Delta H^{2} /\left[\left(\Delta H_{\mathrm{pp}} \sqrt{3} / 2\right)^{2}+\left(H-H_{\mathrm{res}}\right)^{2}\right] \\
& -V_{\text {antisym }}\left(\Delta H_{\mathrm{pp}} \sqrt{3} / 2\right)\left(H-H_{\mathrm{res}}\right) /\left[\left(\Delta H_{\mathrm{pp}} \sqrt{3} / 2\right)^{2}\right. \\
& \left.+\left(H-H_{\mathrm{res}}\right)^{2}\right],
\end{aligned}
$$

where $\Delta H_{\mathrm{pp}}$ is the peak-to-peak linewidth and $H_{\mathrm{res}}$ is the resonance field. $V_{\text {sym }}$ can be produced by the ISHE combined with any contributions from the anisotropic magnetoresistance (AMR) effect—planar Hall effect (PHE) part—and the anomalous Nernst effect (ANE) $[23,24] . V_{\text {antisym }}$ generally results from the anomalous Hall effect $[23,24]$. The lineardependences of $V_{\text {sym }}$ and $V_{\text {antisym }}$ with $P$ (and thus with $h_{r f}^{2}$ ) were demonstrated [Fig. 1(c)]. In addition to these measurements, absorption spectra were measured [Fig. 1(d)]. Lock-in detection was used to enhance the signal-to-noise ratio. Data were fitted using a Lorentzian derivative to determine $\Delta H_{\mathrm{pp}}$ and $H_{\text {res }}$.

\section{RESULTS AND DISCUSSIONS}

\section{A. Temperature-dependence}

The experiments and data analysis described above were conducted at $T$ ranging between 50 and $300 \mathrm{~K}$ (Fig. 2). The key novel result of our article is that, for $\mathrm{NiFe}, V_{\text {sym }}$ displays a nonmonotonous $T$ dependence. From Fig. 2(a), $V_{\text {sym }}$ can be seen to flip sign upon reversal of $H$. This observation agrees with the time-reversal symmetry properties of the ISHE [6,7]. The PHE, which is odd in $H$, can be omitted. Figures 2(b) and 2(c) also show that the nonmonotonous $T$ dependence of $V$ is not related to $V_{\text {antisym }}$ nor to the possible PHE, as deduced from the AMR [25] evolution obtained separately for $H=1 \mathrm{kOe}$ using standard four-point measurements [26]. The nonmonotonous $T$ dependence of $V$ was also independent of $\Delta H_{\mathrm{pp}}$ versus $T$, which was monotonous [Fig. 2(c)] 

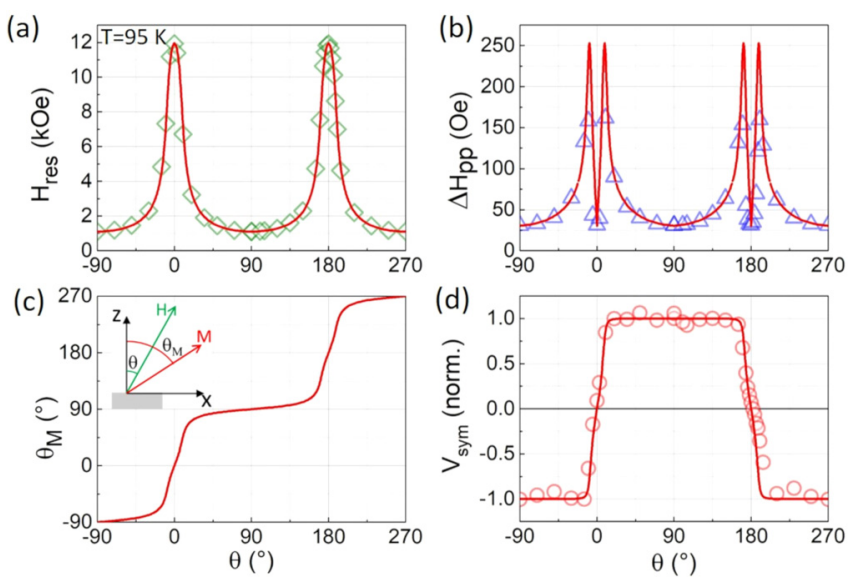

FIG. 3. $\theta$ dependences of (a) $H_{\text {res }}$, (b) $\Delta H_{\mathrm{pp}}$, (c) the tilt in magnetization $\theta_{\mathrm{M}}$, and (d) $V_{\text {sym }}$. Data measured at $95 \mathrm{~K}$ for a $\mathrm{Si} / \mathrm{SiO}_{2} / / \mathrm{Cu}(6) / \mathrm{NiFe}(8) / \mathrm{Cu}(3) / \mathrm{Al}(2) \mathrm{Ox}(\mathrm{nm})$ stack. The lines were obtained using models described in the text.

[27,28]. The total Gilbert damping was determined using the following equation: $\alpha=\left(\Delta H_{\mathrm{pp}}-\Delta H_{\mathrm{pp} 0}\right) \sqrt{3}|\gamma| /(4 \pi f)$. Inhomogeneous broadening $\left(\Delta H_{\mathrm{pp} 0}\right)$ due to spatial variations in the magnetic properties could reasonably be neglected when making estimations at $9.6 \mathrm{GHz}$, since $T$-invariant values of just a few oersted were found using similar samples and a broadband setup. The gyromagnetic ratio was determined by fitting data related to the $f$ dependence of $H_{\text {res }}$ at $300 \mathrm{~K}$, and a reasonable value of $\gamma=18.8 \mathrm{MHz} \mathrm{Oe}^{-1}$ was obtained. In line with [29], a potential $T$-dependent change in the direction of anisotropy could also be ruled out from the behavior of $H_{\text {res }}$ versus $T$ [Fig. 2(d)]. Data were satisfactorily described using the usual Kittel formula [30].

\section{B. Angular-dependence}

To gain further insight into the origins of $V_{\text {sym }}$, we performed angular $(\theta)$-dependent measurements for $T=$ $95 \mathrm{~K}$ (maximal signal). Experimental data were compared to numerical calculations [Fig. 3(a)]. The following set of equations describing equilibrium conditions was considered $[10,25,31]: \quad 2 H_{\text {res }} \sin \left(\theta-\theta_{M}\right)+4 \pi M_{S} \sin \left(2 \theta_{M}\right)=0 ; \quad$ and $(\omega / \gamma)^{2}=\left[H_{\mathrm{res}} \cos \left(\theta-\theta_{M}\right)-4 \pi M_{S} \cos (2 \theta)\right]\left[H_{\mathrm{res}} \cos (\theta-\right.$ $\left.\left.\theta_{M}\right)-4 \pi M_{S} \cos ^{2}\left(\theta_{M}\right)\right]$, where $M_{S}$ is the saturation magnetization and $\theta_{\mathrm{M}}$ is the tilt in $\boldsymbol{M}$. Numerical minimization returned $M_{S}=700 \mathrm{emu} \mathrm{cm} \mathrm{cm}^{-3}$ and $\gamma=$ 18.5 $\mathrm{MHz} \mathrm{Oe}^{-1}$. The expression $\Delta H_{\mathrm{pp}}=(2 / \sqrt{3}) \alpha(\omega / \gamma) /$ $\cos \left(\theta-\theta_{M}\right)+\left|d H_{\text {res }} / d \theta\right| \Delta \theta$ [25,31] was used to describe the data shown in Fig. 3(b). Numerical minimization returned $\alpha=0.008$, and $\Delta \theta=0.25^{\circ}$. The $\theta$ dependence of $\theta_{\mathrm{M}}$ was also determined from the calculations and is plotted in Fig. 3(c). The related transverse voltage resulting from the ISHE was calculated by applying the following theoretical expression [10]: $V_{\text {sym }}$ (norm.) $=\sin \left(\theta_{M}\right)\left[4 \pi M_{S} \gamma \sin ^{2}\left(\theta_{M}\right)+\right.$ $\left.\sqrt{\left(4 \pi M_{S} \gamma \sin ^{2}\left(\theta_{M}\right)\right)^{2}+4 \omega^{2}}\right] /\left[\left(4 \pi M_{S} \gamma \sin ^{2}\left(\theta_{M}\right)\right)^{2}+4 \omega^{2}\right]$.

The correspondence between experimental data and theoretical predictions [Fig. 3(d)] indicates that the ISHE may be the main effect influencing the $T$ dependence observed.
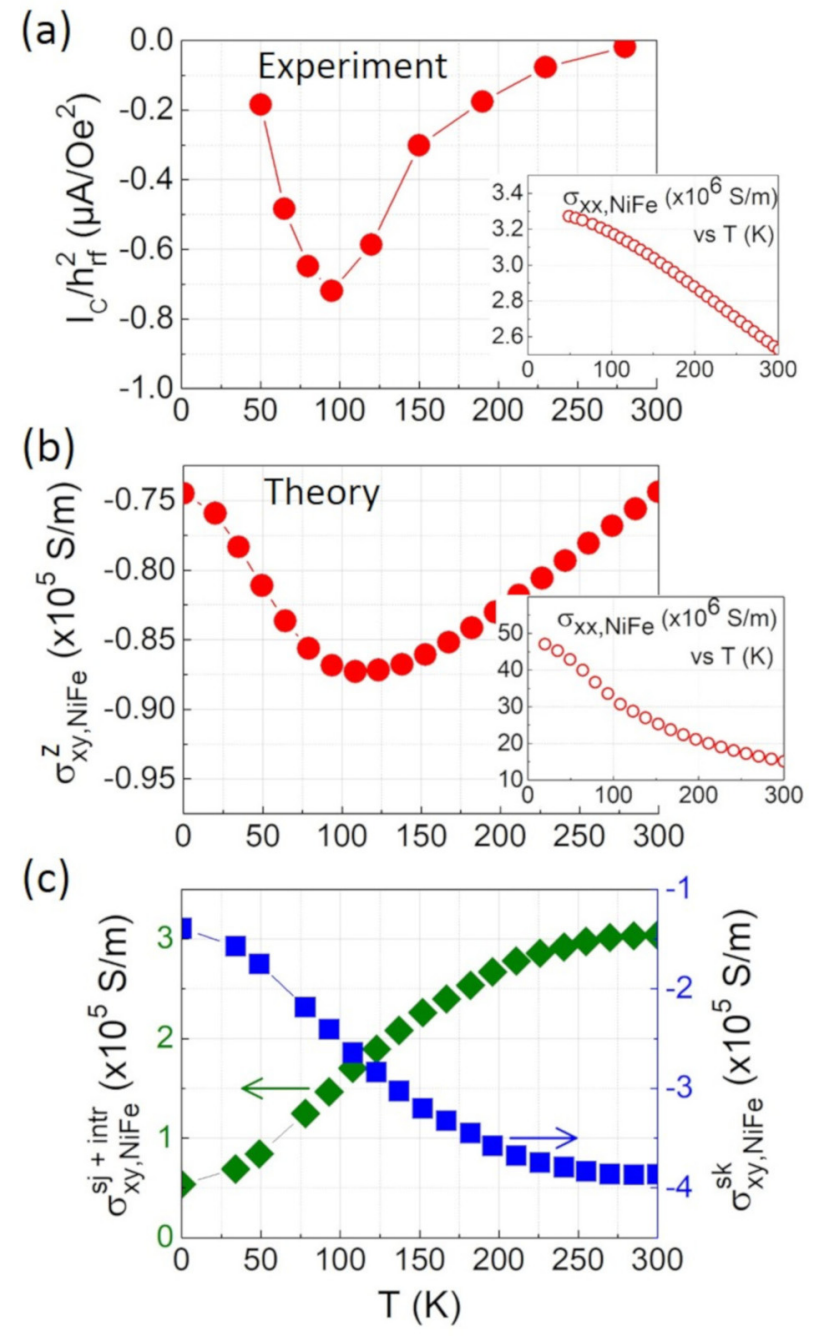

FIG. 4. (a) $T$ dependence of the charge current $\left(I_{C}\right)$ generated by spin-charge conversion in an $\mathrm{Si} / \mathrm{SiO}_{2} / / \mathrm{Cu}(6) / \mathrm{NiFe}(8) / \mathrm{Cu}(3) /$ $\mathrm{Al}(2) \mathrm{Ox}$ (nm) stack. (Inset) $T$ dependence of the NiFe layer's longitudinal conductivity $\left(\sigma_{x x, \mathrm{NiFe}}\right)$ obtained independently using fourpoint measurements. (b) $T$ dependence of the spin Hall conductivity of bulk NiFe $\left(\sigma_{x y, \mathrm{NiFe}}^{z}\right)$ determined from first-principles calculations. (Inset) $T$ dependence calculated for $\sigma_{x x, \mathrm{NiFe}}$. (c) Skew scattering $\left(\sigma_{x y, \mathrm{NiFe}}^{\mathrm{sk}}\right)$ and side jump plus intrinsic $\left(\sigma_{x y, \mathrm{NiFe}}^{\mathrm{sj}+\mathrm{intr}}\right)$ contributions.

\section{Theory}

We then compared the charge current deduced from our experimental data [Fig. 4(a)]: $I_{C}=\left[V_{\text {sym, } \theta=-90^{\circ}}-\right.$ $\left.\left.V_{\text {sym, } \theta=90^{\circ}}\right)\right] /(2 R)$, where $R$ is the resistance of the slab, to first-principles calculations of spin Hall conductivity [Fig. 4(b)]. Readers should note that data are discussed in terms of charge current to take the experimental contributions of the $\mathrm{Cu}$ layer and contacts into account. When performing calculations, the thin film was considered a bulk material. For these calculations, the spin-polarized relativistic Korringa-Kohn-Rostoker (SPR-KKR) code was used [32-34]. In this code, the linear response Kubo formalism was implemented in a fully relativistic multiple-scattering KKR Green function method. Thermal effects were modeled by considering electron scattering due to lattice vibration to 
be the dominant effect, because application of $H$ in the ferromagnetic resonance (FMR) experiments quenched spin fluctuations. The $T$ dependence of transversal spin Hall conductivity $\left(\sigma_{x y, \mathrm{NiFe}}^{z}\right)$ is in satisfactory qualitative agreement with the experimental findings, showing a non-monotonous behavior with a minimum around $T=100 \mathrm{~K}$. To gain more insight into the origins of the effect, we further disentangled the skew scattering $\left(\sigma_{x y, \mathrm{NiFe}}^{\mathrm{sk}}\right)$ and side jump plus intrinsic $\left(\sigma_{x y, \mathrm{NiFe}}^{\mathrm{sj}}\right)$ contributions, based on an approach using scaling behavior $[6,7,35]$. The following equation was considered: $\sigma_{x y, \mathrm{NiFe}}^{z}=\sigma_{x y, \mathrm{NiFe}}^{\mathrm{sk}}+\sigma_{x y, \mathrm{NiFe}}^{\mathrm{sj}+\mathrm{intr}}=\sigma_{x x, \mathrm{NiFe}} \mathrm{S}+\sigma_{x y, \mathrm{NiFe}}^{\mathrm{sj}+\mathrm{intr}}$, where $S$ is the skewness factor. For every $T$ tested, $\sigma_{x x, \mathrm{NiFe}}$ was varied by changing the composition of the alloy over a range from $\mathrm{Ni}_{85} \mathrm{Fe}_{15}$ to $\mathrm{Ni}_{70} \mathrm{Fe}_{30}$. S was subsequently determined from plots of $\sigma_{x y, \mathrm{NiFe}}^{z}$ versus $\sigma_{x x, \mathrm{NiFe}}$. The two contributions, $\sigma_{x y, \mathrm{NiFe}}^{s k}=\sigma_{x x, \mathrm{NiFe}} \mathrm{S}$ and $\sigma_{x y, \mathrm{NiFe}}^{\mathrm{sj} \text { intr }}$, were then plotted [Fig. 4(c)] to determine the $\mathrm{Ni}_{81} \mathrm{Fe}_{19}$ composition. The nonmonotonous $T$ dependence of $\sigma_{x y, \mathrm{NiFe}}^{z}$ could clearly be ascribed to the fact that the skew scattering and the side-jump plus intrinsic contributions have opposite signs and similar amplitudes. These results can be phenomenologically understood in the light of the resonant scattering model that takes split impurity levels into consideration [36,37]. By inserting the scattering phase shift of $\mathrm{Fe}$ in $\mathrm{Ni}$, returned by the SPR-KKR code, into the equations for spin Hall proposed in Ref. [36], we determined the ratio between the skew scattering and sidejump contributions to be around -1.2 . The same trend of opposing signs and similar amplitudes was observed. This finding also seems to infer that the intrinsic ISHE is negligible in permalloy.

\section{Stacking-dependence}

Interestingly, similar sets of experimental $T$ dependences for $I_{C}$ were obtained whatever the material in contact with the permalloy: $\mathrm{SiO}_{2}, \mathrm{MgO}, \mathrm{AlOx}$ oxides, $\mathrm{Cu}$, and $\mathrm{Pt}$ metals (Fig. 5). This observation further confirms the bulk origin of the effect. Figure 5(a) corresponds to the reference sample, where the NiFe layer was encapsulated between two metallic $\mathrm{Cu}$ layers. The data in Fig. 5(b) show that replacing a $\mathrm{Cu} / \mathrm{NiFe}$ interface by an $\mathrm{SiO}_{2} / \mathrm{NiFe}$ interface does not alter the $T$-dependent profile for the charge current. For Figs. 5(c) and $5(\mathrm{~d})$, the $\mathrm{NiFe} / \mathrm{Cu}$ and $\mathrm{Cu} / \mathrm{NiFe}$ interfaces were replaced by interfaces with $\mathrm{AlOx}$ and $\mathrm{MgO}$. These samples were grown in a different sputter machine, which could explain the discrepancy in signal amplitude. In addition, we cannot exclude the possibility that the quality of the NiFe stack grown on $\mathrm{AlOx}$ and $\mathrm{MgO}$ differ from that of the $\mathrm{NiFe}$ stack grown on $\mathrm{SiO}_{2}$ or $\mathrm{Cu}$. Nevertheless, the nonmonotonous $T$ dependence of $I_{C}$ was qualitatively similar for this set of samples. Figure 5(e) corresponds to a $\mathrm{Pt} / \mathrm{NiFe} / \mathrm{Cu}$ stack. With this sample, spin-charge conversion in Pt shifts the signal downwards. Figure 5(f) corresponds to the $\mathrm{Cu} / \mathrm{NiFe} / \mathrm{Pt}$ stack, where opposing spin-charge conversion occurs in the $\mathrm{NiFe}$ and Pt layers. Substituting a $\mathrm{Cu} / \mathrm{IrMn}$ layer for the Pt spincharge converter induced a similar effect, thus confirming the findings. Note that for this latter case, $\mathrm{Cu}$ was used to avoid exchange bias coupling between the $\mathrm{NiFe}$ and IrMn layers. The samples including buffer and capping Pt layers, and the

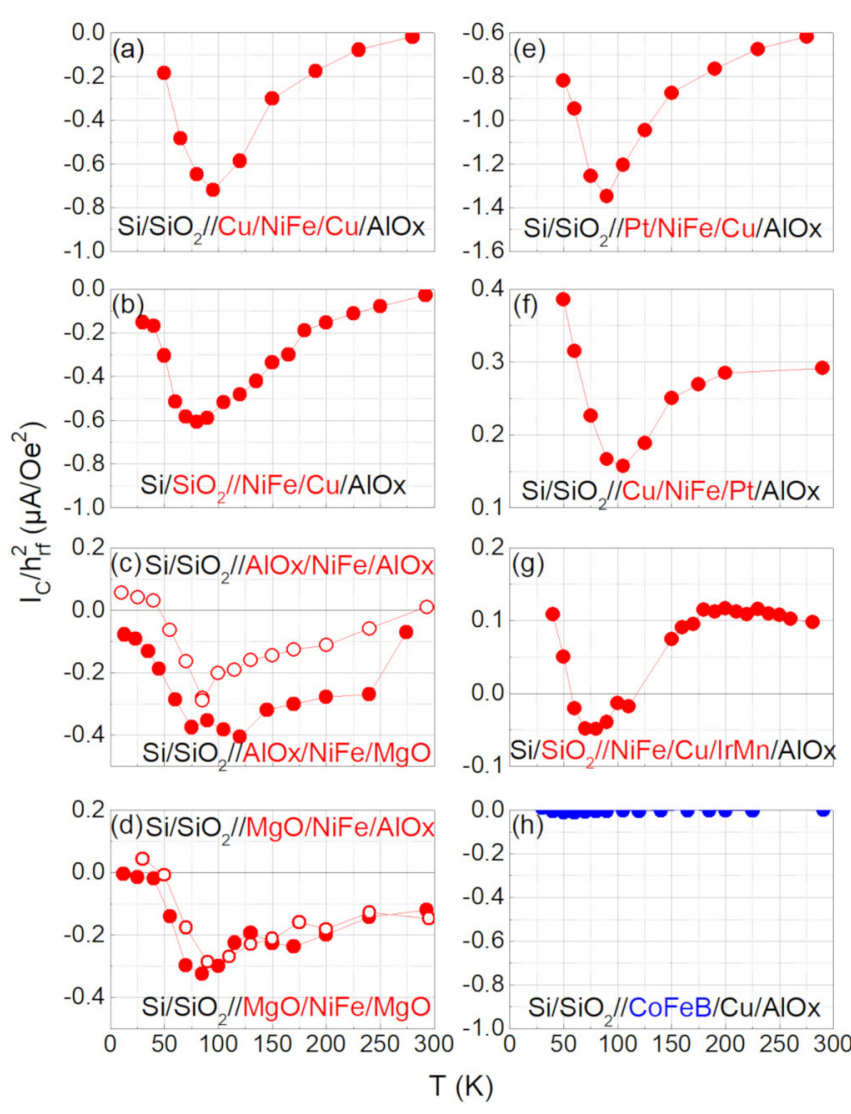

FIG. 5. (a) $T$ dependence of $I_{C}$ generated in several stacks deposited on $\mathrm{Si} / \mathrm{SiO}_{2} / /$ substrates. The compositions were (a) //Cu(6)/ $\mathrm{NiFe}(8) / \mathrm{Cu}(3) / \mathrm{Al}(2) \mathrm{Ox}$, (b) $/ / \mathrm{NiFe}(8) / \mathrm{Cu}(3) / \mathrm{Al}(2) \mathrm{Ox}$, (c) $/ / \mathrm{AlOx}$ (20)/NiFe(16)/AlOx(20) and //AlOx(20)/NiFe(16)/MgO(20), (d) $/ / \mathrm{MgO}(20) / \mathrm{NiFe}(16) / \mathrm{AlOx}(20)$ and $/ / \mathrm{MgO}(20) / \mathrm{NiFe}(16) /$ $\mathrm{MgO}(20)$, (e) $/ / \mathrm{Pt}(10) / \mathrm{NiFe}(8) / \mathrm{Cu}(6) / \mathrm{Al}(2) \mathrm{Ox}$, (f) $/ / \mathrm{Cu}(6) / \mathrm{NiFe}(8) /$ $\mathrm{Pt}(10) / \mathrm{Al}(2) \mathrm{Ox}, \quad(\mathrm{g}) / / \mathrm{NiFe}(8) / \mathrm{Cu}(3) / \mathrm{IrMn}(1.5) / \mathrm{Al}(2) \mathrm{Ox}$, and (h) $/ / \mathrm{CoFeB}(8) / \mathrm{Cu}(3) / \mathrm{Al}(2) \mathrm{Ox}(\mathrm{nm})$.

important observations and conclusions that can be drawn from these measurements, will be discussed further below. Finally, Fig. 5(g) shows that the effect was absent when the $\mathrm{NiFe}$ was replaced by a $\mathrm{CoFeB}$ layer.

While we cannot definitively exclude that MCP [14,18-20] also occurs in our samples, our experimental and theoretical data indicated that our observations mainly relate to the selfinduced ISHE. In particular, theory and the findings presented in Fig. 5 support a bulk origin of the effect, obviating the need to consider the spatial inversion symmetry which is compulsory for MCP [14,18-20]. The results shown in Fig. 5 also demonstrate that our observations are not linked to the ANE [38-40]. This effect could also generate a transverse charge current due to SOI, and shares the same symmetry as the ISHE. It is known to result from a $T$-gradient building up when maximum power is absorbed by the F. Because the thermal conductivity of the oxides used in our experiments is of the order of $\mathrm{W} \mathrm{m}^{-1} \mathrm{~K}^{-1}$ compared to a few hundred for the metals, significant changes in the amplitude of ANErelated observations is expected. However, our observations were independent of the heat-sinking efficiency of the stack (Fig. 6). These results were also corroborated by the fact 

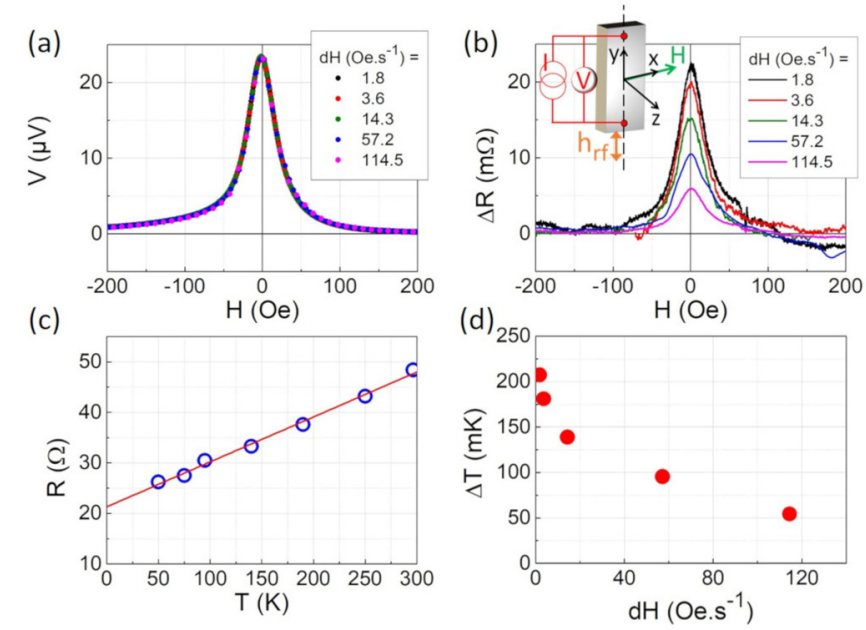

FIG. 6. (a) Representative $V$ vs $H-H_{\text {res }}$ for a $\mathrm{Si} / \mathrm{SiO}_{2} / /$ $\mathrm{Cu}(6) / \mathrm{NiFe}(32) / \mathrm{Cu}(3) / \mathrm{Al}(2) \mathrm{Ox}(\mathrm{nm})$ stack at $T=95 \mathrm{~K}$, with $\theta=$ $+90^{\circ}$ (no bias current). Several field-sweep rates $(\mathrm{dH})$ were used for the magnetic field. (b) The same dependences as in (a) were measured when a bias current of $I=100 \mu \mathrm{A}$ was applied across the sample (see inset). The change in the sample's resistance was estimated after removing the off-resonance voltage, as follows: $\left.\Delta R=\left[V_{\text {with bias current }}-V_{\text {without bias current }}\right)\right] / I$. (c) $T$ dependence of the off-resonance sample's resistance, measured independently. (d) $d H$ dependence of the increase in temperature of the sample at resonance $(\Delta T)$, deduced from (b) and (c).

that the signal observed was independent of the field-sweep rate, over the range accessible with our experimental setup [Fig. 6(a)] [40]. We note that this behavior remains valid despite an estimated temperature increase of up to about $200 \mathrm{mK}$ [Fig. 6(d)], due to the absorption of microwave power by the sample at resonance.

\section{E. Direction of the spin current}

We will now comment on the direction of the self-induced current $\left(J_{S \text {, self }}\right)($ Fig. 7$)$. A reference layer of Pt was added

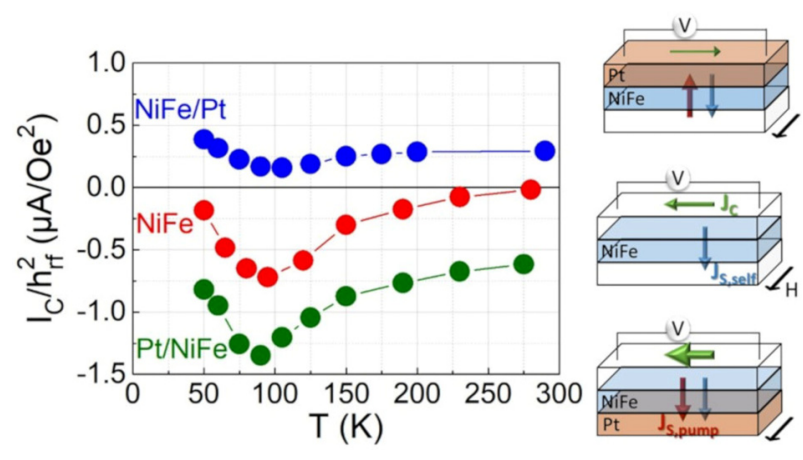

FIG. 7. (a) $T$ dependence of $I_{C}$ generated in $\mathrm{Si} / \mathrm{SiO}_{2} / / \mathrm{Pt}(10) /$ $\mathrm{NiFe}(8) / \mathrm{Cu}(6) / \mathrm{Al}(2) \mathrm{Ox}$ (buffer $\mathrm{Pt}), \mathrm{Si} / \mathrm{SiO}_{2} / / \mathrm{Cu}(6) / \mathrm{NiFe}(8) /$ $\mathrm{Pt}(10) / \mathrm{Al}(2) \mathrm{Ox}$ (capping $\mathrm{Pt}$ ), and $\mathrm{Si} / \mathrm{SiO}_{2} / / \mathrm{Cu}(6) / \mathrm{NiFe}(8) /$ $\mathrm{Cu}(3) / \mathrm{Al}(2) \mathrm{Ox}(\mathrm{nm})$ stacks. (b) Representations of the spin and charge currents in the stacks. to the stack, either as a buffer or as a capping layer. In this case, spin-charge conversions produced by ISHE in the Pt and NiFe layers contribute concurrently to the total experimentally probed $I_{C}$. The Pt layer has a positive spin Hall angle $\left(\Theta_{\text {ISHE,Pt }}\right)$. For sufficiently thick layers, $V_{\text {sym }}$ generated in Pt relates to $\Theta_{\text {ISHE,Pt }} l_{\text {sf,Pt }}$ because $\Theta_{\text {ISHE,Pt }}$ is known to be mostly intrinsic [41-43]. $\Theta_{\text {ISHE,Pt }} l_{\text {sf,Pt }}$ and $V_{\text {sym }}$ are therefore $T$-independent. Furthermore, $V_{\text {sym }}$ in Pt flips sign when the stacking order or field are reversed [6,7]. Given this fact, and considering the electrical connections in our setup, a buffer Pt layer pumps a spin current $\left(J_{S, \mathrm{Pt}}\right)$ toward the substrate and returns a negative (positive) value of $V_{\text {sym }}$ for a field angle $\theta=-90^{\circ}\left(90^{\circ}\right)$, resulting in a negative value of $\left.I_{C}=\left[V_{\text {sym, } \theta=-90^{\circ}}-V_{\text {sym, } \theta=90^{\circ}}\right)\right] /(2 R)$. Conversely, when a capping Pt layer is included, a positive value of $I_{C}$ is returned. The NiFe layer also has a positive Hall angle [13]. The findings presented in Fig. 7 therefore indicate that, with regards to spin current direction, the NiFe layer behaves similarly to a buffer Pt layer, as it induces a negative $I_{C}$. In this scenario, spin and subsequent charge currents in the Pt and $\mathrm{NiFe}$ layers add up for the buffer Pt layer case, and subtract for the capping case (inset of Fig. 7). Similar to previous experiments [13], the spin current may be generated as a result of asymmetric spin-dependent scattering across the NiFe film, possibly due to nonhomogeneous film properties across its thickness and to subsequent asymmetric spin relaxation at the various interfaces. From these data, at $T \sim 95 \mathrm{~K}$, the self-induced conversion of the $\mathrm{NiFe}$ can be as efficient as that observed with Pt. We also note that although spin-charge conversion in $\mathrm{NiFe}$ is inefficient close to $300 \mathrm{~K}$ and only relates to ISHE in the Pt layer, self-induced spin diffusion still occurs. This effect creates asymmetry in the conversion and may contribute to the observed difference in $I_{C}$ measured at $300 \mathrm{~K}$ due to the inversion of the Pt growth order. Inverting the growth order also modifies the electric properties of the $\mathrm{Pt}$ layer and interfaces. For example, we measured a resistivity of $\sigma_{x x, \mathrm{Pt}}=4 \times 10^{6}$ and $5 \times 10^{6} \mathrm{~S} \mathrm{~m}^{-1}$ for the capping and buffer layers, respectively, corresponding to reasonable $l_{\mathrm{sf}, \mathrm{Pt}}$ values $(\sim 3-4 \mathrm{~nm})$ for the spin diffusion length [43]. We note that, if $J_{S \text {, self }}$ were omitted, $\Theta_{\text {ISHE,Pt }} l_{\text {sf,Pt }}$ at $300 \mathrm{~K}$ could be calculated using the following equation: $\Theta_{\mathrm{ISHE}, \mathrm{Pt}} l_{\mathrm{sf}, \mathrm{Pt}}=$ $\frac{I_{C}}{h_{\mathrm{rf}}{ }^{2}} \frac{1}{w \tanh \left[t_{\mathrm{Pt}} /\left(2 l_{\mathrm{sf}, \mathrm{Pt}}\right)\right]} \frac{8 \pi \alpha^{2}}{2 e g_{r}^{\uparrow} \gamma^{2}} \frac{\left(4 \pi M_{S} \gamma\right)^{2}+4 \omega^{2}}{4 \pi M_{S} \gamma+\sqrt{\left(4 \pi M_{S} \gamma\right)^{2}+4 \omega^{2}}}$, where the spin mixing conductance is calculated from $g_{r}^{\uparrow \downarrow}=2 \sqrt{3} \pi M_{S} \gamma$ $t_{\mathrm{NiFe}} \Delta H^{\text {pump }} /\left(g \mu_{B} \omega\right), \quad$ with $\quad \Delta H^{\text {pump }}=\left(\Delta H_{\mathrm{pp}, \mathrm{NiFe} / \mathrm{Pt}}-\right.$ $\left.\Delta H_{\mathrm{pp}, \mathrm{NiFe}}\right)$ for the capping Pt layer case and $\Delta H^{\mathrm{pump}}=$ $\left(\Delta H_{\mathrm{pp}, \mathrm{Pt} / \mathrm{NiFe}}-\Delta H_{\mathrm{pp}, \mathrm{NiFe}}\right)$ for the buffer layer [10]. Using the parameters measured separately, $M_{S}=700 \mathrm{emu} \mathrm{cm} \mathrm{cm}^{-3}$, $\gamma=18.5 \mathrm{MHz} \mathrm{Oe}^{-1}, \Delta H_{\mathrm{pp}, \mathrm{NiFe} / \mathrm{Pt}}=57 \mathrm{Oe}, \Delta H_{\mathrm{pp}, \mathrm{Pt} / \mathrm{NiFe}}=$ $48 \mathrm{Oe}, \Delta H_{\mathrm{pp}, \mathrm{NiFe}}=29 \mathrm{Oe}$, we determined $g_{r}^{\uparrow \downarrow}=27$ and $18 \mathrm{~nm}^{-2}$ for the capping and buffer $\mathrm{Pt}$ layer cases, respectively. The $\tanh \left[t_{\mathrm{Pt}} /\left(2 l_{\mathrm{sf}, \mathrm{Pt}}\right)\right]$ can be approximated to 1 . When further considering the values of $I_{C} / h_{\mathrm{rf}}{ }^{2}$ returned from the data in Fig. 7 at $300 \mathrm{~K}$, we calculated $\Theta_{\text {ISHE,Pt }} l_{\text {sf,Pt }}=0.23$ and $0.52 \mathrm{~nm}$ for the capping and buffer $\mathrm{Pt}$ layer cases, respectively. These data give the expected order of magnitude for $\mathrm{Pt}$ [43]. The discrepancy between the two values tends to confirm that $J_{S \text {, self }}$ should not be neglected when determining $\Theta_{\text {ISHE,Pt }}$ [12], although stacking order-dependent interfacial spin asymmetry 

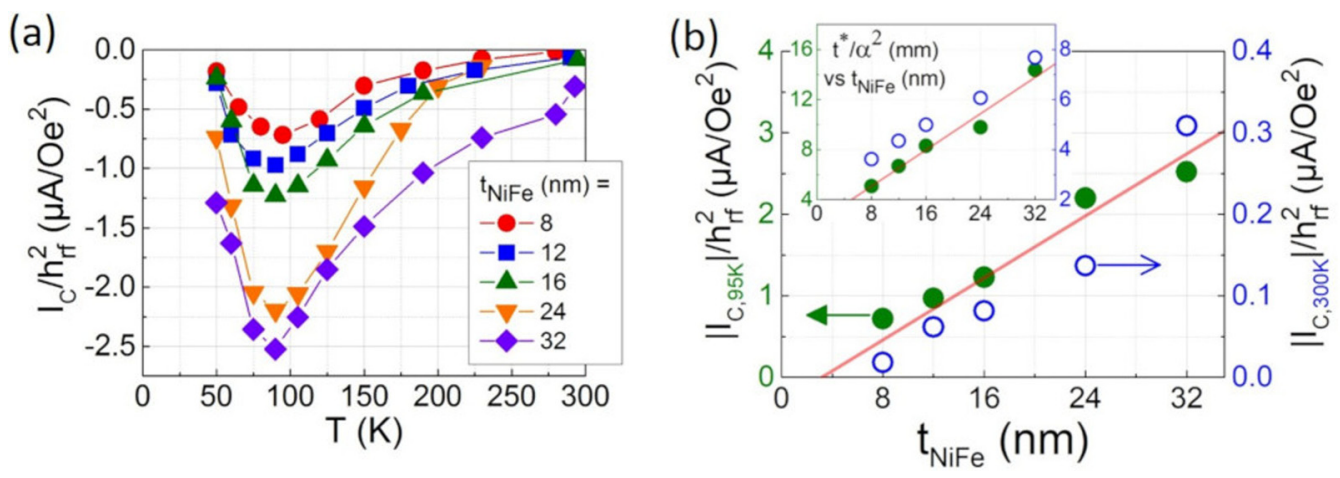

FIG. 8. (a) $T$ dependence of $I_{C}$ measured in $\mathrm{Si} / \mathrm{SiO}_{2} / / \mathrm{Cu}(6) / \mathrm{NiFe}\left(t_{\mathrm{NiFe}}=8 ; 12 ; 16 ; 24 ; 32\right) / \mathrm{Cu}(3) / \mathrm{Al}(2) \mathrm{Ox}(\mathrm{nm})$ stacks. (b) $\mathrm{NiFe}$ thickness dependence of $I_{C}$ measured at 95 and $300 \mathrm{~K}$. (Inset) Corresponding thickness dependences of $t^{*} / \alpha^{2}$.

and spin-flip scattering parameters may also contribute [43].

\section{F. Thickness-dependence}

We finally considered how the effect was affected by the $\mathrm{NiFe}$ layer thickness. The contributions of the $\mathrm{Cu}$ layer and contacts were taken into account by considering the charge current rather than just the voltage drop, thus it was relevant to compare several NiFe thicknesses. We found that the position of maximum conversion $I_{C, 95 \mathrm{~K}}$ was thickness-independent [Fig. 8(a)]. This observation is also in agreement with the bulk origin of the effect. We further observed that the amplitude of $I_{C, 95 \mathrm{~K}}$ showed a similar thickness dependence to $I_{C, 300 \mathrm{~K}}$ [Fig. 8(b)]. The thickness dependence of $I_{C}$ relates to $t^{*} / \alpha^{2}$, where $1 / \alpha^{2}$ accounts for the spin pumping efficiency, and $t^{*}$ describes the thickness dependence of the spin-charge conversion efficiency [10]. The former parameter was found to increase with thickness in a linear fashion. This behavior is due to the decreasing role played by interfaces, and the subsequent decrease of $\alpha$ for thick layers [44]. For the conversion efficiency, in this case, the spin sink is also the $\mathrm{NiFe}$ spin current generator. Considering that the spin current is due to asymmetric spin relaxation at the various interfaces, we get a situation similar to the case of a spin-sink receiving the spin current from a third party and can thus consider that $t^{*}=l_{\mathrm{sf}, \mathrm{NiFe}} \tanh \left[t_{\mathrm{NiFe}} /\left(2 l_{\mathrm{sf}, \mathrm{NiFe}}\right)\right][10] . l_{\mathrm{sf}, \mathrm{NiFe}}$ was estimated by combining our measurements of longitudinal conductivity in the following relation [45]: $l_{\mathrm{sf}, \mathrm{NiFe}}=0.91 \sigma_{x x, \mathrm{NiFe}} \times 10^{-12}$. The values calculated for $l_{\mathrm{sf}, \mathrm{NiFe}}$ at $T=100 \mathrm{~K}$ ranged between $2.9 \mathrm{~nm}$ for 8 -nm-thick NiFe films to $5.3 \mathrm{~nm}$ for the 32-nm-thick film, in agreement with [46]. Plotting $t^{*} / \alpha^{2}$ versus $T$ [inset of Fig. 8(b)] revealed a nearly linear behavior, corroborating the results of the thickness dependence of $I_{C}$.

\section{CONCLUSION}

In conclusion, the main contribution of this paper is that it presents systematic evidence of a self-induced ISHE in FMR experiments. Our findings were supported by distinct sets of $T$-, thickness-, angular-, and stack-dependent experimental data encompassing the main features of the self-induced ISHE. The experimental findings were corroborated by firstprinciples calculations. Most importantly, similar amplitudes but opposite signs for the bulk skew scattering and the sidejump plus intrinsic contributions to the $T$-dependent spin Hall conductivity in permalloy could explain why the SOI-related transverse voltage was observed to display non-monotonous $T$ dependence. The findings from this study contribute to our understanding of a previously overlooked and incompletely understood effect. The results further indicate that self-induced conversion within the ferromagnet can be as efficient as that recorded with noble metals such as $\mathrm{Pt}$, and thus needs to be carefully considered when investigating SO-related effects in materials destined for use in spintronics.

\section{ACKNOWLEDGMENTS}

We acknowledge financial support from the French national research agency (ANR) (Grant No. ANR-15-CE240015-01) and KAUST (Grant No. OSR-2015-CRG4-2626). We also thank O. Sipr for fruitful discussions and $\mathbf{M}$. Gallagher-Gambarelli for critical reading of the manuscript.
[1] Series on Semiconductor Science and Technology, edited by S. Maekawa and S. O. Valenzuela, E. Saitoh, and T. Kimura (Oxford University Press, Oxford, 2012).

[2] Y. Otani, M. Shiraishi, A. Oiwa, E. Saitoh, and S. Murakami, Nat. Phys. 13, 829 (2017).

[3] A. V. Chumak, A. D. Karenowska, A. A. Serga, and B. Hillebrands, Nat. Phys. 11, 453 (2015).
[4] S. Maekawa, H. Adachi, K. Uchida, J. Ieda, and E. Saitoh, J. Phys. Soc. Japan 82, 102002 (2013).

[5] F. Hellman, A. Hoffmann, Y. Tserkovnyak, G. S. D. Beach, E.E. Fullerton, C. Leighton, A. H. MacDonald, D. C. Ralph, D. A. Arena, H. A. Durr, P. Fischer, J. Grollier, J. P. Heremans, T. Jungwirth, A. V. Kimmel, B. Kooplans, I. N. Krivorotov, S. J. May, A. K. Petford-Long, J. M. Rondinelli, N. Samarth, I. 
K. Schuller, A. N. Slavin, M. D. Stiles, O. Tchernyshyov, A. Thiaville, and B. L. Zink, Rev. Mod. Phys. 89, 025006 (2017).

[6] A. Hoffmann, IEEE Trans. Magn. 49, 5172 (2013).

[7] J. Sinova, S. O. Valenzuela, J. Wunderlich, C. H. Back, and T. Jungwirth, Rev. Mod. Phys. 87, 1213 (2015).

[8] Y. Tserkovnyak, A. Brataas, G. E. W. Bauer, and B. I. Halperin, Rev. Mod. Phys. 77, 1375 (2005).

[9] K. Ando, Semicond. Sci. Technol. 29, 043002 (2014).

[10] K. Ando, S. Takahashi, J. Ieda, Y. Kajiwara, H. Nakayama, T. Yoshino, K. Harii, Y. Fujikawa, M. Matsuo, S. Maekawa, and E. Saitoh, J. Appl. Phys. 109, 103913 (2011).

[11] B. F. Miao, S. Y. Huang, D. Qu, and C. L. Chien, Phys. Rev. Lett. 111, 066602 (2013).

[12] A. Azevedo, O. A. Santos, R. O. Cunha, R. Rodriguez-Suarez, and S. M. Rezende, Appl. Phys. Lett. 104, 152408 (2014).

[13] A. Tsukahara, Y. Ando, Y. Kitamura, H. Emoto, E. Shikoh, M. P. Delmo, T. Shinjo, and M. Shiraishi, Phys. Rev. B 89, 235317 (2014).

[14] C. Ciccarelli, K. M. D. Hals, A. Irvine, V. Novak, Y. Tserkovnyak, H. Kurebayashi, A. Brataas, and A. Ferguson, Nat. Nanotechnol. 10, 50 (2014).

[15] K. Kanagawa, Y. Teki, and E. Shikoh, AIP Adv. 8, 055910 (2018).

[16] Y. Omori, E. Sagasta, Y. Niimi, M. Gradhand, L. E. Hueso, F. Casanova, and Y. C. Otani, Phys. Rev. B 99, 014403 (2019).

[17] W. Wang, T. Wang, V. P. Amin, Y. Wang, A. Radhakrishnan, A. Davidson, S. R. Allen, T. J. Silva, H. Ohldag, D. Balzar, B. L. Zink, P. M. Haney, J. Q. Xiao, D. G. Cahill, V. O. Lorenz, and X. Fan, Nat. Nanotechnol. 14, 819 (2019).

[18] A. Azevedo, R. O. Cunha, F. Estrada, O. Alves Santos, J. B. S. Mendes, L. H. Vilela-Leão, R. L. Rodríguez-Suárez, and S. M. Rezende, Phys. Rev. B 92, 024402 (2015).

[19] K. M. D. Hals and A. Brataas, Phys. Rev. B 91, 214401 (2015).

[20] F. Freimuth, S. Blügel, and Y. Mokrousov, Phys. Rev. B 92, 064415 (2015).

[21] K. Klein, B. Hauer, B. Stoib, M. Trautwein, S. Matich, H. Huebl, O. Astakhov, F. Finger, R. Bittl, M. Stutzmann, and M. S. Brandt, Rev. Sci. Instrum. 84, 103911 (2013).

[22] S.-I. Kim, M.-S. Seo, and S.-y. Park, J. Appl. Phys. 115, 17 C501 (2014).

[23] M. Harder, Y. Gui, and C. M. Hu, Phys. Rep. 661, 1 (2016).

[24] R. Iguchi and E. Saitoh, J. Phys. Soc. Japan 86, 011003 (2017).

[25] J.-C. Rojas-Sánchez, M. Cubukcu, A. Jain, C. Vergnaud, C. Portemont, C. Ducruet, A. Barski, A. Marty, L. Vila, J.-P.
Attané, E. Augendre, G. Desfonds, S. Gambarelli, H. Jaffrès, J.-M. George, and M. Jamet, Phys. Rev. B 88, 064403 (2013).

[26] I. Miccoli, F. Edler, H. Pfnur, and C. Tegenkamp, J. Phys. Condens. Matter 27, 223201 (2015).

[27] K. Gilmore, Y. U. Idzerda, and M. D. Stiles, Phys. Rev. Lett. 99, 027204 (2007).

[28] L. Frangou, S. Oyarzun, S. Auffret, L. Vila, S. Gambarelli, and V. Baltz, Phys. Rev. Lett. 116, 077203 (2016).

[29] J. F. Sierra, V. V. Pryadun, F. G. Aliev, S. E. Russek, M. GarcíaHernández, E. Snoeck, and V. V. Metlushko, Appl. Phys. Lett. 93, 172510 (2008).

[30] C. Kittel, Phys. Rev. 73, 155 (1948).

[31] J. Smit and H. G. Beljers, Philips Res. Rep. 10, 113 (1955).

[32] H. Ebert, D. Ködderitzsch, and J. Minár, Reports Prog. Phys. 74, 096501 (2011).

[33] H. Ebert, J. Braun, D. Ködderitzsch, and S. Mankovsky, Phys. Rev. B 93, 075145 (2016).

[34] The Munich SPR-KKR package, Http://Olymp.Cup.UniMuenchen.de/Ak/Ebert/SPRKKR version 6.3 (2012).

[35] Y. Tian, L. Ye, and X. Jin, Phys. Rev. Lett. 103, 087206 (2009).

[36] A. Fert and P. M. Levy, Phys. Rev. Lett. 106, 157208 (2011).

[37] P. M. Levy, H. Yang, M. Chshiev, and A. Fert, Phys. Rev. B 88, 214432 (2013).

[38] S. Y. Huang, W. G. Wang, S. F. Lee, J. Kwo, and C. L. Chien, Phys. Rev. Lett. 107, 216604 (2011).

[39] M. Weiler, M. Althammer, F. D. Czeschka, H. Huebl, M. S. Wagner, M. Opel, I.-M. Imort, G. Reiss, A. Thomas, R. Gross, and S. T. B. Goennenwein, Phys. Rev. Lett. 108, 106602 (2012).

[40] P. Noël, M. Cosset-Cheneau, V. Haspot, V. Maurel, C. Lombard, A. Barthelemy, L. Vila, and J.-P. Attané, arXiv:1905.00771.

[41] M. Isasa, E. Villamor, L. E. Hueso, M. Gradhand, and F. Casanova, Phys. Rev. B 91, 024402 (2015).

[42] Y. Wang, P. Deorani, X. Qiu, J. H. Kwon, and H. Yang, Appl. Phys. Lett. 105, 152412 (2014).

[43] J.-C. Rojas-Sánchez, N. Reyren, P. Laczkowski, W. Savero, J.P. Attané, C. Deranlot, M. Jamet, J.-M. George, L. Vila, and H. Jaffrès, Phys. Rev. Lett. 112, 106602 (2014).

[44] A. Ghosh, S. Auffret, U. Ebels, and W. E. Bailey, Phys. Rev. Lett. 109, 127202 (2012).

[45] E. Sagasta, Y. Omori, M. Isasa, Y. Otani, L. E. Hueso, and F. Casanova, Appl. Phys. Lett. 111, 082407 (2017).

[46] G. Zahnd, L. Vila, V. T. Pham, M. Cosset-Cheneau, W. Lim, A. Brenac, P. Laczkowski, A. Marty, and J.-P. Attané, Phys. Rev. B 98, 174414 (2018). 